NURC: CELEBRATING 50 YEARS OF

\title{
The Interaction of \\ Marine Mammals
and Active Sonar
}

BY KENDRA RYAN

Over the last 50 years, international

public concern regarding human impact

on the environment has increased

drastically. Specific to the maritime domain, attention has more acutely focused during the last ten years on the temporal and spatial relationships of marine mammals' strandings to the use of active sonar. The NATO Undersea Research Centre (NURC) has been at the forefront of research regarding the interaction of marine mammals and active sonar. In 1999, NURC established one of the first research programs in the world to address this topic, the Sound Ocean Living Marine Resources (SOLMAR) program, now known as the Marine Mammal Risk Mitigation (MMRM) program. With the participation of numerous international partners, marine mammal risk mitigation policies, procedures, and technologies were implemented, and continue to be developed, to minimize the potential impact on marine mammals.

\section{FOUNDING OF SOLMAR}

From May 12-15, 1996, NURC (formerly known as SACLANT Undersea Research Centre, SACLANTCEN) conducted the Shallow Water Acoustic Classification (SWAC '96) research cruise using low- and mid-frequency (600- $\mathrm{Hz}$ and 3-kHz, respectively) active sonars off the coast of Greece (SACLANT Undersea Research Centre, 1998). Concurrently, 12-13 Cuvier's beaked whales (Ziphius cavirostris) stranded along the coast of Kyparissiakos Gulf, Greece. NURC hosted a bioacoustics panel comprised of subject experts shortly after Frantzis (1998) hypothesized an association between the SWAC experiment and the stranding event. The panel concluded that an acoustic link was neither clearly established nor eliminated as a direct or indirect cause of the May 1996 stranding (SACLANT Undersea Research Centre, 1998). Despite the nondefinitive conclusion, the panel recommended that environmental assessment procedures be implemented as soon as possible with a view to recommending suitable mitigation and monitoring protocols. In addition, the panel determined that additional research was needed to determine biological and behavioral characteristics of marine mammals. The findings and recommendations of the 1998 Bioacoustics Panel prompted the creation of the SOLMAR program.

\section{SOLMAR OBJECTIVES}

(1999-2006)

The SOLMAR program was a multinational, multidisciplinary research project with the objective of developing the tools and/or procedures with which an experimenter can ensure there are no

Lt. Kendra Ryan (USN) (ryan@nurc.nato. int) was Marine Mammal Risk Mitigation Project Officer, NATO Undersea Research Centre, La Spezia, Italy, from January 2007 to May 2008. 

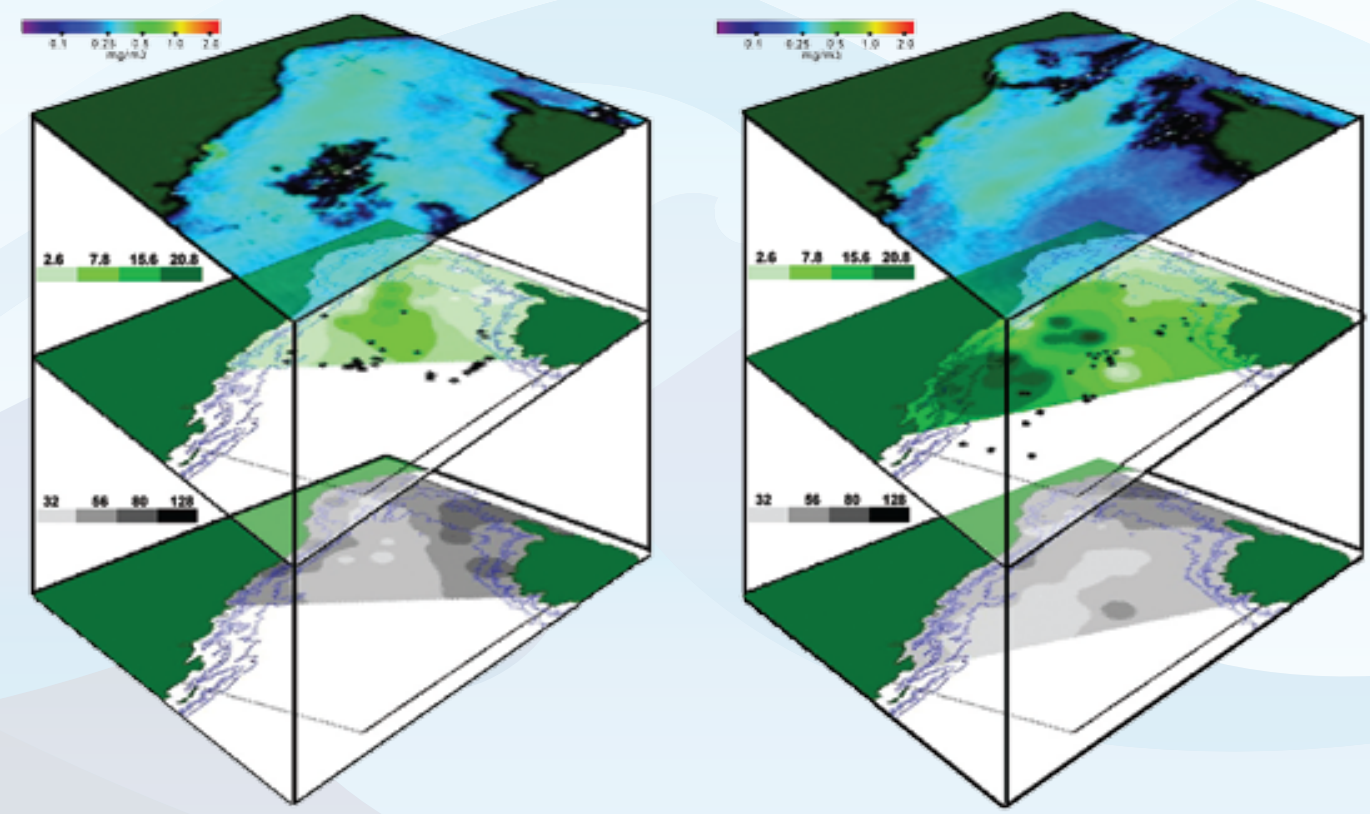

Figure 1. Sirena ' 99 (left) and Sirena '00 (right) Sea WIFS chlorophyll $a\left(\mathrm{mg} \mathrm{m}^{-3}\right)$ in the upper layer, chlorophyll $a$ maximum ( $\mathrm{mg} \mathrm{m}^{-3}$ ) with fin whale visual sightings in the middle layer, and the depth of the maximum values of chlorophyll $a$ in the lower layer. SACLANT Undersea Research Centre, 2001

marine mammals near a sonar source prior to and during its use (D'Amico et al., 2003b). At the inception of the program, little was known about the regions of high and low cetacean density within the areas where NURC experiments were conducted. The first tasks of the project were therefore to develop:

- A network of scientists with knowledge of marine mammal distribution, behavior, bioacoustics, and sonars

- A database containing all documents and data related to marine mammal distribution, behavior, and associated bioacoustics information

- A risk mitigation instruction based on available knowledge that contained policy and protocols to ensure that active acoustic research was planned and performed in an environmentally sensitive manner (SACLANT Undersea Research Centre, 2004) Upon completion of these tasks, the project shifted focus to development of: - A database containing information on marine mammal populations in the Mediterranean Sea and environmental parameters in relationship to these population densities

- Acoustic and visual tools to detect cetaceans

To accomplish these last two objectives, a series of sea trials, entitled SIRENA, were conducted.

\section{SIRENA}

The SOLMAR program conducted SIRENA sea trials annually in the Ligurian Sea from 1999 to 2003. Bounded by Italy, mainland France, and Corsica, the Ligurian Sea was designated the first International Marine Sanctuary in the Mediterranean Sea and was the common habitat of eight cetacean species. The NATO research vessel, $\mathrm{R} / \mathrm{V}$ Alliance, was the primary platform for conducting visual and acoustic monitoring of cetaceans. Oceanographic measurements, including temperature, salinity, nutrients (silicates and ammonias), fluorescence, turbidity, and phytoplankton (chlorophyll), were gathered concurrently from an Italian navy hydrographic vessel and from various other vessels provided by partner organizations. Sea surface temperature, surface currents, surface roughness, and ocean color were collected in real time at NURC using satellite remote sensing.

\section{SIRENA RESULTS}

The SIRENA cruises provided a vast quantity of data that enabled scientists to answer some of the primary questions regarding Mediterranean Sea cetaceans and their habitat preferences. During SIRENA '99, a total of 869 individuals was observed during 146 marine mammal sighting events (D'Amico et al., 2000). Analysis of the data from 1999 and those from SIRENA '00 showed that the fin whale (Balaenoptera physalus) and sperm whale (Physeter macrocephalus) generally preferred cold, deep, nutrientrich portions of the basin (Figure 1; 
SACLANT Undersea Research Centre, 2001) while the Cuvier's beaked whales preferred a submarine canyon (the Genoa Canyon) where there is a frontal influence and steep bathymetry (D'Amico et al., 2003b).

Also during SIRENA '00, a sperm whale was tagged with a noninvasive digital recording device (Johnson and Tyack, 2003) to gather data regarding the whale's diving behavior and vocalization activity. The data indicated that the average depth of this sperm whale's foraging dives was $900 \mathrm{~m}$. At this foraging depth, the acoustic behavior of the sperm whale changed, and the data supported the hypothesis that sperm whales echolocate on prey at a constant horizontal layer (Zimmer et al., 2003). Such data advance knowledge of normal sperm whale behavior so that behavioral responses to anthropogenic activities may be better understood.

The objectives of the SIRENA '01 sea trial were to continue collection of oceanographic and species data for population of the developing database and to evaluate techniques for effective risk mitigation (SACLANT Undersea

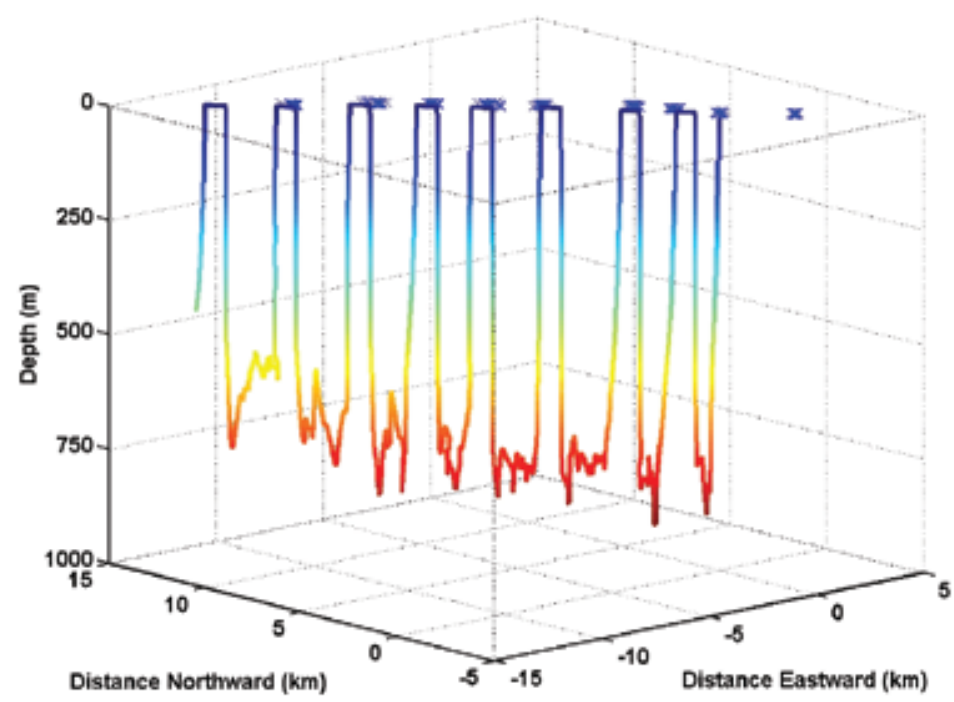

Figure 2. Three-dimensional track of a tagged sperm whale. Surface positions marked with an " $\mathrm{x}$ " indicate a visual sighting. Tyack and Zimmer, 2002

combined with visual and passive acoustic methods to enable three-dimensional tracking of the whale's movements and foraging events (Figure 2; Tyack and Zimmer, 2002). Analysis of this information supported conclusions regarding sperm whale habitat preference that were derived from previous SIRENA cruise data (SACLANT Undersea Research

\section{(NURC) has been at the forefront of} research regarding the interaction of marine mammals and active sonar.

Research Centre, 2000). During the cruise, tag data (e.g., vocalizations, dive depths, and whale orientation) transmitted from a digital recording device attached to a sperm whale were
Centre, 2002). In addition, visual sightings and acoustic data from a towed array contributed to the population of the marine-mammal-sighting database.

During SIRENA '02 and '03, the application of digital recording devices to sperm whales continued, and operations included exploring the feasibility of a whale-finder sonar. Using defined protocols, the feasibility test involved controlled transmission of an active sound source in the vicinity of tagged animals. Analysis of the passive acoustic data confirmed that sperm whales produce multipulse clicks centered around $15 \mathrm{kHz}$, with source levels of more than $230 \mathrm{~dB}$ re $1 \mu \mathrm{Pa} @ 1 \mathrm{~m}$, and a narrow, forward-oriented sonar beam (Møhl et al., 2003; Zimmer et al., 2005b). Additionally, in 2002, the SIRENA sea trial focused on determining the specific habitat of the Cuvier's beaked whale. Based on Cuvier's sighting data from previous years, a survey of the Genoa Canyon $\left(\sim 10,600 \mathrm{~km}^{2}\right)$ was undertaken. Analysis of the oceanographic and presence data determined that all Cuvier's sightings were in water depths greater than $1000 \mathrm{~m}$. These areas may provide a 
preferential habitat (one with an abundant food source) due to the oceanographic conditions that result from the interaction of the Ligurian current and the canyon walls. In addition, the canyon environment can form traps for suspended particulate matter containing high biomass (D’Amico et al., 2001).

Prior to 2004, the sperm whale was the animal of interest for tagging due to its ease of acoustic and visual detection as compared to the beaked whale. Although valuable information regarding tagging and exposure protocols was obtained from the sperm whale experiments, still little was known regarding the particular behavior of the Cuvier's beaked whale. However, in 2004, analyses of data from tags simultaneously applied to two Cuvier's baked whales resulted in the first estimates of the source level, directivity index, and spectral properties of Cuvier's beaked whale clicks (Figure 3; Zimmer et al., 2005a).

As a result of the SOLMAR program, a thorough database was constructed that contains visual sightings during the SIRENA trials, additional sighting and stranding data from collaborating organizations, and historical data gathered from literature reviews. This database contains 5463 sighting data points and 4300 stranding data points, making it one of the most extensive data sets on marine mammals in the Mediterranean Sea.

\section{MARINE MAMMAL RISK MITIGATION}

Although some of the initial questions regarding the habitat and characteristics of Cuvier's beaked whales remained unanswered, ongoing operational and research requirements necessitated the
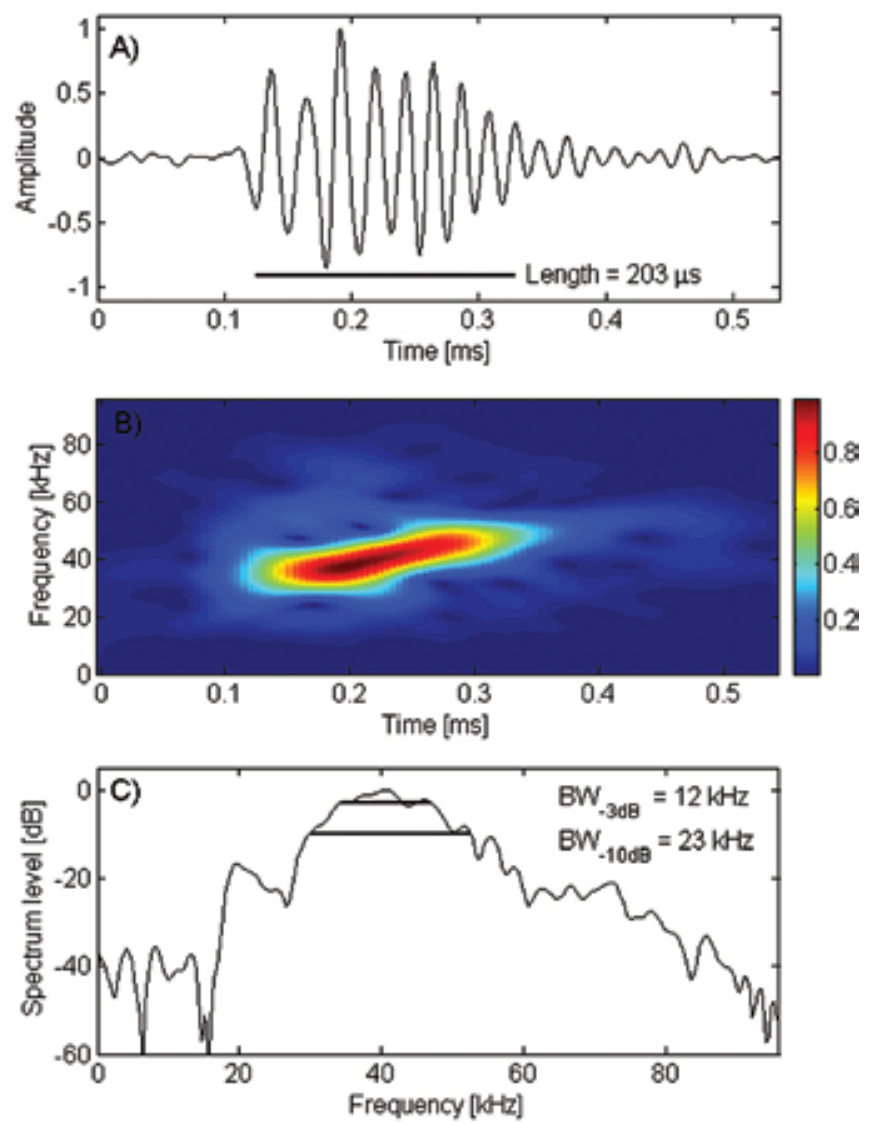

Figure 3. Sample of Z. cavirostris click as received by the tag of a conspecific whale. The top panel shows the time series, the middle panel shows the spectrogram, and the bottom panel shows the relative power spectrum. Zimmer et al., 2005 a

design and implementation of a marine mammal risk mitigation package based on the information acquired during the previous years of SIRENA sea trials.

This package provided researchers and military commanders with aids to assess the potential for a given area of the sea to contain beaked whales and other marine mammals that may be impacted by the use of active sonar. The package also provided mitigation techniques and protocols to be implemented during the use of active sonar. This shift in project objectives correlated with a change in the project title from SOLMAR to the Marine Mammal Risk Mitigation (MMRM) project.

Components of the package included passive acoustic monitors, a predictive habitat model, a sound propagation model, a Web site with training aides, and a guiding policy for the use of active sonar in experiments at NURC. The passive acoustic monitors implemented in the toolkit were the result of evolving methods and technologies used in each successive year of the SIRENA cruises. In the early years of the project, Cuvier's beaked whales' vocalizations were unknown. Thus, methodologies 
that had previously recorded other cetaceans in low-frequency $(<1 \mathrm{kHz})$ and mid-frequency $(1-10 \mathrm{kHz})$ ranges were implemented. Instrumentation used included a 32-element passive array with real-time beamforming in frequencies from $750 \mathrm{~Hz}$ to $4.1 \mathrm{kHz}$ during SIRENA '99 and SIRENA '00, and the use of a 128-element array to the predictive habitat model, a tool to determine the presence or absence of species of interest in a certain geographical area. The basis of the habitat model is the quantification of species and their relationship to environmental factors using sighting and environmental data from past SIRENA cruises. From these data, distinct environmental variables

...marine mammal risk mitigation policies, procedures, and technologies were

implemented...to minimize the potential impact on marine mammals.

provide frequency coverage from $200 \mathrm{~Hz}$ to $16 \mathrm{kHz}$ (Zimmer et. al., 2003). These arrays were suitable for detecting the broadband echolocation clicks of sperm whales; however, the range of the devices was insufficient to detect the Cuvier's beaked whale. It was not until beaked whale clicks were recorded on a digital recording tag that the frequency range in which these animals vocalize was revealed. The following year, analysis of data obtained from digital recording tags applied to two Cuvier's beaked whales revealed vocalizations with a center frequency of $42 \mathrm{kHz}$ (Zimmer et al., 2005a). New arrays and compact passive acoustic monitors (CPAMS) that include this higher frequency will be deployed in SIRENA '08 in an effort to acquire more data on the vocalization behavior of Cuvier's beaked whales.

Another component of the marine mammal risk mitigation package is were determined to correlate with a particular species (Azzelino et al., 2001). The model contains predictor variables for fin whales, sperm whales, Cuvier's beaked whales, and striped dolphins. Such a model offers the possibility of focusing field activities in much smaller areas and developing mitigation options, such as avoiding active sonar operations in areas where there is high probability of encountering sensitive species.

The third component of the marine mammal risk mitigation package is a sound propagation tool. Prior to conducting an experiment, characteristics of active sources that will be used in the experiment and the geographic area of the experiment are input to the model to predict propagation loss. From this prediction, visual monitoring ranges are estimated. During the experiment, if animals are sighted within these monitoring ranges, active sonar use must be modified until the animals are no longer detected in the area. This model and additional information about the marine protected areas and the most common species of the Mediterranean Sea are available on the project Web site (http:// www.solmar.nurc.nato.int).

\section{ENVIRONMENTAL POLICY}

One of the initial objectives of the SOLMAR project was to develop a risk mitigation instruction that contained policies and protocols to ensure that active acoustic research was planned and performed without negative impact to human divers and marine mammals (http://solmar.nurc.nato.int). This instruction, entitled "NATO Undersea Research Centre Human Diver and Marine Mammal Risk Mitigation Rules" (NATO Undersea Research Centre, 2006), was created shortly after the 1998 Bioacoustics Panel convened. It was originally based on knowledge available at the time, but is continually updated by the MMRM program to reflect the latest research in this field. The instruction is multilayered, with the main points stating that all Centre sea trials must perform an environmental scoping study prior to any experiment, that a maximum received sound-pressure level or energy-density level must not be exceeded during the experiment, and that risk-mitigation techniques must be applied during the experiment. In addition, the instruction was modified to include reference to the Marine Mammal Crisis Action Team, now called the Human Diver and Marine Mammal Incident Action Team. The purpose of this team is to respond to marine mammal stranding incidents or diver accidents that may be temporally or spa- 
tially associated to Centre activities, to assist national authorities analyzing the event, and to assess the oceanographic conditions related to the incident. Since the initial policy was developed in 1999, no known marine mammal strandings have occurred in spatial or temporal concurrence to Centre experiments.

\section{INTERNATIONAL}

\section{COLLABORATION}

The proactive approach adopted by NURC includes engaging academia, government, and environmental organizations into its MMRM program. Over 20 participants from nine countries have contributed resources including software, technologies, data, and personnel to the planning, execution, and analyses of sea trials. In 2005 and 2007, NURC organized two intergovernmental conferences entitled "The Effects of Sound in the Ocean on Marine Mammals." Over 175 individuals from 11 countries participated in these events, which provided a forum for military personnel and government-funded scientists to discuss the status of current research regarding the potential impacts of sonar on marine mammals. In addition, current mitigation methods employed by national navies were shared. These conferences provided opportunities for discussion of coordinated efforts and future collaborations to ensure that maximum benefit results from limited research funding and to eliminate duplicative efforts.

\section{CONCLUSION}

NURC has maintained a robust science program for the last 10 years designed to address the topic of marine mammals and anthropogenic noise. The SOLMAR program and the following MMRM program collaborated with academia, government, and environmental organizations of partner nations to collect data and generate guiding principles for the safe conduct of active sonar experiments in the presence of marine mammals. By adopting a proactive approach, NURC has not only been able to successfully conduct its program of work, but the Centre is also now recognized as a leader in this area and a good steward of the environment.

\section{ACKNOWLEDGEMENTS}

All approaches to the animals for tagging were made following the conditions of NMFS Scientific Research Permit 981-1578-00. This research was approved by the Woods Hole Oceanographic Institution Animal Care and Use Committee. 마

\section{REFERENCES}

Azzellino, A., J.F. Borsani, A. D’Amico, D. Demer, D. McGehee, N. Portunato, and V. Teloni. 2001. A G.I.S. integrated database to investigate cetacean distribution in the Ligurian Sanctuary. (Sirena ' 99 and '00 operations). In Abstracts of the 14th Biennial Conference on the Biology of Marine Mammals, Vancouver, Canada, November 28-December 3, 2001.

D’Amico, A., F. Mineur, A. Alvarez, C. Mori, M. Podestà, N. Portunato, M. Ballardini, and B. Nani. 2001. Oceanographic correlations with the distribution of Cuvier's Beaked Whales (Ziphius cavirostris) in the Ligurian Sea. Poster presented at the 14th Biennial Conference on the Biology of Marine Mammals, Vancouver, Canada, November 28-December 3, 2001.

D’Amico, A., A. Azellino, C. Misic, M. Podesta, M. Carron, and N. Portunato. 2003a. Presentation at the Symposium on Environmental Consequences of Underwater Sound, San Antonio, Texas, May 12-16, 2003.

D’Amico, A., A. Bergamasco, P. Zanasca, S. Carniel, E. Nacini, N. Portunato, V. Teloni, C. Mori, and R. Barbanti. 2003b. Qualitative correlation of marine mammals with physical and biological parameters in the Ligurian Sea. IEEE Journal of Oceanic Engineering 28:29-43.

Frantzis, A. 1998. Does acoustic testing strand whales? Nature 392:29.

Johnson, M.P., and P.L. Tyack. 2003. A digital acoustic recording tag for measuring the response of wild marine mammals to sound. IEEE Journal of Oceanic Engineering 28:3-12.

Møhl, B., M. Wahlberg, P.T. Madsen, A. Heerfordt, and A. Lund. 2003. The monopulsed nature of sperm whale clicks. Journal of the Acoustical Society of America 114:1,143-1,154.

NATO Undersea Research Centre. 2006. Human Diver and Marine Mammal Risk Mitigation Rules and Procedures. NURC Special Publication 2006-008, 21 pages. Available online at: http://solmar.nurc. nato.int/ (accessed May 21, 2008).

SACLANT Undersea Research Centre. 1998. Bioacoustics Panel and Marine Mammal Environmental and Mitigation Procedures Panel. A. D'Amico, ed. NATO Undersea Research Centre, Italy, SACLANTCEN M-133, 84 pp.

SACLANT Undersea Research Centre. 2000. Scientific Programme of Work 2001. NATO Undersea Research Centre, Italy, 98 pp.

SACLANT Undersea Research Centre. 2001. Annual Progress Report 2001. NATO Undersea Research Centre, Italy, 140 pp.

SACLANT Undersea Research Centre. 2002. Annual Progress Report 2002. NATO Undersea Research Centre, Italy, AE-2001, 145 pp.

SACLANT Undersea Research Centre. 2004. Annual Progress Report 2003. NATO Undersea Research Centre, Italy, AE-2003, $211 \mathrm{pp}$.

Tyack, P., and W.M.X Zimmer. 2002. Presentation at the Undersea Defence Technology Europe conference, Italy, June 19, 2002.

Zimmer, W.M.X., M.P. Johnson, A. D’Amico, and P.L. Tyack. 2003. Combining data from a multisensory tag and passive sonar to determine the diving behavior of a sperm whale (Physeter macrocephalus). IEEE Journal of Oceanic Engineering 28:13-29.

Zimmer, W.M.X., M.P. Johnson, P.T. Madsen, and P.L. Tyack. 2005a. Echolocation clicks of free-ranging Cuvier's beaked whales (Ziphius cavirostris). Journal of the Acoustical Society of America. 117:3,919-3,927.

Zimmer, W.M.X., P.L. Tyack, M.P. Johnson, and P.T. Madsen. 2005b. Three-dimensional beam pattern of regular sperm whale clicks confirms bent-horn hypothesis. Journal of the Acoustical Society of America 117:1,473-1,485. 